\title{
Asymptotic approximations for vibrational modes of helices
}

\author{
T. Tarnopolskaya* $\quad$ F.R. de Hoog*
}

(Received 7 August 2000)

\begin{abstract}
The free vibrations in the plane normal to the helical axis are studied under the assumption that the helical pitch is small. Asymptotic approximations for eigenvalues and eigenfunctions are derived for both small and large numbers of helical turns. The analytic approximations reveal interesting features of helix vibrations and the connection between the vibrational modes of a helix and the flexural modes of a curved beam. Comparison with numerical calculations shows that the
\end{abstract}

${ }^{*}$ CSIRO Mathematical and Information Sciences, Canberra, Australia

${ }^{0}$ See http://anziamj . austms.org. au/V42/CTAC99/Tarn for this article and ancillary services, (C) Austral. Mathematical Soc. 2000. Published 27 Nov 2000. 
approximations derived cover with sufficient accuracy a wide range of number of helical turns.

\section{Contents}

1 Introduction

C1400

2 Governing equations

C1401

3 Approximations at small number of helical turns

C1403

4 Approximations at large number of helical turns

C1406

4.1 Approximation for eigenvalues

$\mathrm{C} 1406$

4.2 Mode shapes at large opening angle

$\mathrm{C} 1410$

5 Numerical results and discussion

C1413

6 Conclusions

C1416

References

C1418 


\section{Introduction}

The vibrations of helices have important engineering applications and continue to attract the attention of researchers $[1,2,3,4,5,6]$. Three-dimensional equations for the vibrations of helices are generally too difficult to solve analytically and natural frequencies are usually calculated by various numerical techniques. The importance of simplified models that provide a deep insight into different effects inherent in helix vibrations has long been recognised $[2,3,4]$.

A simplified model presented in this paper considers a helix as a planar uniformly curved beam vibrating in the plane of beam curvature and is different from published simplified models [2, 3, 4]. Such a model gives an adequate approximation to the vibrations of a helix provided that the helical pitch is small compared with its diameter and that motion parallel to the helical axis is neglected. We start with flexural modes of a moderately curved planar beam and then follow their development as the non-dimensional curvature of the beam increases to arbitrarily large values. A similar approach has been used in the authors' earlier papers[7, 8] for beams with relatively small curvature and revealed many interesting features of mode transition accompanying an increase in beam curvature. Using this approach, it has proved possible to reveal the transformation of modes that occurs with increase in number of helical turns and the association of the modes of a helix with the modes of a curved beam. 
The analysis of the present paper consists of two parts:

1. analysis of vibrational behaviour for a small number of helical turns;

2. analysis of vibrational behaviour for an arbitrarily large number of helical turns.

In both cases, simple analytic approximations for non-dimensional eigenvalue and mode shape are obtained. An interesting feature of the vibrational behaviour for small numbers of helical turns, revealed by the analysis, is the occurrence of a minimum of the non-dimensional eigenvalue at a value of the opening angle that is a function of the mode number. This minimum relates to the minimum of strain energy. The analysis also reveals that there are two different types of vibrational behaviour for a large number of helical turns, characterised by quite different mode shapes. These modes, and their succession in the frequency spectrum, are examined. Numerical calculations illustrating the vibrational behaviour at different ranges of opening angle and confirming the validity of the analytical approximations are presented.

\section{Governing equations}

As mentioned in the introduction, the equations for in-plane vibrations of a uniformly curved beam with large opening angle can be used to approximate 
the vibrations of a helix, provided that the helical pitch is small compared with its diameter and that the motion in the direction of the helical axis is neglected. The equations for the free in-plane vibrations of a uniformly curved beam in non-dimensional form can be written as [7]

$$
\begin{aligned}
\left(u^{\prime}-\alpha v\right)^{\prime}+\epsilon \Lambda u & =0, \\
-\epsilon\left(\alpha^{4} v+2 \alpha^{2} v^{\prime \prime}+v^{\prime \prime \prime \prime}\right)+\left(u^{\prime}-\alpha v\right) \alpha+\epsilon \Lambda v & =0,
\end{aligned}
$$

where $u$ and $v$ are the non-dimensional tangential and normal displacements, $\alpha$ is the opening angle, which is the ratio of beam length $l$ to its radius of curvature $R, \Lambda$ is a non-dimensional eigenvalue and $\epsilon$ is a slenderness parameter of the beam, defined by $\Lambda=\rho l^{2} \omega^{2} /(\epsilon E), \epsilon=h^{2} /\left(12 l^{2}\right)$, where $h$ is the beam thickness, $\rho$ is the density, $E$ is Young's modulus and $\omega$ is the frequency. Primes denote differentiation with respect to the non-dimensional co-ordinate $\bar{s}=s / l$ measured along the beam centreline. For the sake of clarity, it is assumed that the beam has rectangular cross-section and that the ends of the beam are clamped, so that

$$
\begin{array}{ccc}
u=0 & \text { at } & \bar{s}=0,1, \\
v=v^{\prime}=0 \quad \text { at } & \bar{s}=0,1 .
\end{array}
$$

In what follows, we take advantage of the fact that when $\epsilon$ is small and the opening angle $\alpha$ is sufficiently large, the equations (1-4) can be approximated by the equation of inextensional vibrations [9]

$$
v^{\prime \prime \prime \prime \prime \prime \prime}+2 \alpha^{2} v^{\prime \prime \prime \prime}+\left(\alpha^{4}-\Lambda\right) v^{\prime \prime}+\Lambda v \alpha^{2}=0 .
$$


with boundary conditions

$$
v=v^{\prime}=v^{\prime \prime \prime \prime \prime}+2 \alpha^{2} v^{\prime \prime \prime}=0 \quad \text { at } \quad \bar{s}=0,1 .
$$

\section{Approximations at small number of helical turns}

In has been shown in [8] that after the sharp increase in eigenvalues and transformation of mode shapes that accompanies an increase in beam curvature and occurs at small values of the beam opening angle, a stage follows where there is virtually no change in mode shape with further increase in beam curvature. This gives us the chance to try to obtain an estimate for the eigenvalues from Raileigh's principle, provided that a reasonable approximation for mode shape is known. In the case of the modes symmetric in $v$, the approximation for the transverse displacements of inextensional modes can be obtained from equations $(5,6)$ in the limit $\alpha \rightarrow 0$ and is given, up to a multiplicative constant, by

$$
\begin{aligned}
v= & \sinh \frac{\beta}{2} \cos \left[\beta\left(\bar{s}-\frac{1}{2}\right)\right]+\sin \frac{\beta}{2} \cosh \left[\beta\left(\bar{s}-\frac{1}{2}\right)\right] \\
& -\cosh \frac{\beta}{2} \sin \frac{\beta}{2}-\cos \frac{\beta}{2} \sinh \frac{\beta}{2},
\end{aligned}
$$


where $\beta=\left.\Lambda^{1 / 4}\right|_{\alpha=0}$ and satisfies the equation

$$
\cosh \frac{\beta}{2} \sin \frac{\beta}{2}+\cos \frac{\beta}{2} \sinh \frac{\beta}{2}-\frac{4}{\beta} \sin \frac{\beta}{2} \sinh \frac{\beta}{2}=0 .
$$

The expression for tangential displacements $u$ can be obtained by requiring that the centreline of the beam is unextended (that is, $u^{\prime}-\alpha v=0$ ) and satisfying the boundary condition (3). Up to a multiplicative constant, it is given by

$$
\begin{aligned}
u= & \alpha \int_{0}^{\bar{s}} v d \bar{s}=\frac{\alpha}{\beta}\left\{\sin \frac{\beta}{2} \sinh \left[\beta\left(\bar{s}-\frac{1}{2}\right)\right]\right. \\
& \left.+\sinh \frac{\beta}{2} \sin \left[\beta\left(\bar{s}-\frac{1}{2}\right)\right]+2 \sin \frac{\beta}{2} \sinh \frac{\beta}{2}(1-2 \bar{s})\right\} .
\end{aligned}
$$

The Rayleigh quotient gives

$$
\Lambda=\frac{\int_{0}^{1}\left[\left(v^{\prime}+\alpha u\right)^{\prime}\right]^{2} d \bar{s}}{\int_{0}^{1}\left(u^{2}+v^{2}\right) d \bar{s}}
$$

where the expression in the numerator represents a scaled strain energy. Substituting the expressions for the displacements into (8) gives

$$
\begin{aligned}
\Lambda= & \left\{\left(\beta^{2}+\alpha^{2}\right)^{2}(\sinh \beta+\beta) \sin ^{2} \frac{\beta}{2}+\left(\beta^{2}-\alpha^{2}\right)^{2}(\sin \beta+\beta) \sinh ^{2} \frac{\beta}{2}\right. \\
& \left.-\frac{16}{\beta}\left(\beta^{4}+\alpha^{4}\right) \sin ^{2} \frac{\beta}{2} \sinh ^{2} \frac{\beta}{2}\right\}\left\{( \frac { \alpha } { \beta } ) ^ { 2 } \left[\beta\left(\sinh ^{2} \frac{\beta}{2}-\sin ^{2} \frac{\beta}{2}\right)\right.\right.
\end{aligned}
$$


$\left.-10 \sin \frac{\beta}{2} \sinh \frac{\beta}{2}\left(\cosh \frac{\beta}{2} \sin \frac{\beta}{2}-\cos \frac{\beta}{2} \sinh \frac{\beta}{2}\right)+\frac{8 \beta}{3} \sin ^{2} \frac{\beta}{2} \sinh ^{2} \frac{\beta}{2}\right]$

$\left.+\beta\left(\sinh ^{2} \frac{\beta}{2}+\sin ^{2} \frac{\beta}{2}\right)-\frac{8}{\beta} \sin ^{2} \frac{\beta}{2} \sinh ^{2} \frac{\beta}{2}\right\}^{-1}$,

where allowed $\beta$ values are the roots of equation (7).

It can be seen that the strain energy goes through a minimum. In the case of symmetric modes, the value of the opening angle $\alpha_{*}$ that provides a minimum to the strain energy is given by

$$
\alpha_{*}^{2}=\beta^{2} \frac{(\beta+\sin \beta) \sinh ^{2} \frac{\beta}{2}-(\beta+\sinh \beta) \sin ^{2} \frac{\beta}{2}}{(\beta+\sin \beta) \sinh ^{2} \frac{\beta}{2}+(\beta+\sinh \beta) \sin ^{2} \frac{\beta}{2}-\frac{16}{\beta} \sin ^{2} \frac{\beta}{2} \sinh ^{2} \frac{\beta}{2}} .
$$

For $\beta \geq 2$ this yields $\alpha_{*} \approx \beta \approx 2 \pi n+3 \pi / 2$. One can see that the minima of the non-dimensional eigenvalue for consecutive symmetric modes are spaced $2 \pi$ apart. Because the integral in the denominator of (8) increases with increasing opening angle of the beam, the position of the minimum of the nondimensional eigenvalue $\Lambda$ is shifted toward larger opening angle compared to the position of the minimum of strain energy.

In the case of modes antisymmetric in $v$, the approximation for the eigenvalue can be calculated in a similar manner and is given by

$$
\Lambda=\left\{\left(\beta^{2}+\alpha^{2}\right)^{2}(\sinh \beta-\beta) \cos ^{2} \frac{\beta}{2}-\left(\beta^{2}-\alpha^{2}\right)^{2}(\sin \beta-\beta) \cosh ^{2} \frac{\beta}{2}\right\}
$$




$$
\begin{aligned}
& \times\left\{( \frac { \alpha } { \beta } ) ^ { 2 } \left[-10 \cos \frac{\beta}{2} \cosh \frac{\beta}{2}\left(\cosh \frac{\beta}{2} \sin \frac{\beta}{2}+\cos \frac{\beta}{2} \sinh \frac{\beta}{2}\right)\right.\right. \\
& \left.+\beta\left(\cos ^{2} \frac{\beta}{2}+\cosh ^{2} \frac{\beta}{2}\right)+8 \beta \cos ^{2} \frac{\beta}{2} \cosh ^{2} \frac{\beta}{2}\right] \\
& \left.+\beta\left(\cosh ^{2} \frac{\beta}{2}-\cos ^{2} \frac{\beta}{2}\right)\right\}^{-1} .
\end{aligned}
$$

where $\beta$ is a root of the equation

$$
\cos \frac{\beta}{2} \sinh \frac{\beta}{2}-\cosh \frac{\beta}{2} \sin \frac{\beta}{2}=0 .
$$

The features of the behaviour of the eigenvalue for the modes antisymmetric in $v$ are similar to those for the modes symmetric in $v$.

\section{Approximations at large number of helical turns}

\subsection{Approximation for eigenvalues}

In this section, we obtain approximations for the non-dimensional eigenvalues and mode shapes at large opening angle (or large number of helical turns) 
on the basis of asymptotic analysis of the equation for inextensional vibrations (5). In the following analysis, a parameter $\delta=\sqrt{\Lambda} / \alpha^{2}$ is considered to be small and it is assumed that $\delta=O(1 / \alpha)$. By applying the scaling $\tilde{s}=\alpha \bar{s}$, $\bar{\Lambda}=\Lambda / \alpha^{4}$, equation (5) takes the form

$$
v^{\prime \prime \prime \prime \prime \prime \prime}+2 v^{\prime \prime \prime \prime}+(1-\bar{\Lambda}) v^{\prime \prime}+\bar{\Lambda} v=0
$$

and the characteristic equation can be written as

$$
\gamma^{2}\left(\gamma^{2}+1\right)^{2}=\delta^{2}\left(\gamma^{2}-1\right)
$$

Assuming that $\delta$ is small, the roots of the characteristic equation can be obtained by perturbation analysis of equation (12) and are given by 
Up to order $O\left(\delta^{3}\right)$, this equation can be reduced to

$$
\begin{array}{r}
-\beta_{1} \sin \left(\beta_{1} \alpha / 2\right)\left[\beta_{3}^{3}\left(\beta_{3}^{2}-2\right) \sin \left(\beta_{3} \alpha / 2\right) \cos \left(\beta_{2} \alpha / 2\right)\right. \\
\left.-\beta_{2}^{3}\left(\beta_{2}^{2}-2\right) \cos \left(\beta_{3} \alpha / 2\right) \sin \left(\beta_{2} \alpha / 2\right)\right]=0 .
\end{array}
$$

Obviously, this equation has two families of solutions. The first family is given by the equation

$$
\sin \left(\beta_{1} \alpha / 2\right)=0
$$

for which the solution is

$$
\Lambda_{k}^{\mathrm{I}}=4 \alpha^{2}(\pi k)^{2}, \quad k=1,2, \ldots
$$

The second family is given by the equation

$$
\beta_{3}^{3}\left(\beta_{3}^{2}-2\right) \sin \left(\beta_{3} \alpha / 2\right) \cos \left(\beta_{2} \alpha / 2\right)-\beta_{2}^{3}\left(\beta_{2}^{2}-2\right) \cos \left(\beta_{3} \alpha / 2\right) \sin \left(\beta_{2} \alpha / 2\right)=0,
$$

which can be rewritten, up to the order $O\left(\delta^{2}\right)$, as

$$
\sin \left[\frac{\alpha\left(\beta_{3}-\beta_{2}\right)}{2}\right]=\frac{\alpha\left(\beta_{3}-\beta_{2}\right)}{2} \frac{\sin \alpha}{\alpha} .
$$

Given that $\frac{\sin \alpha}{\alpha}=O(\delta)$, this equation can be solved asymptotically for small $\delta$. Thus, to $\stackrel{\alpha}{O}\left(k \delta^{2}\right)$ we have

$$
\left(\beta_{3}-\beta_{2}\right) \alpha / 2=k \pi\left[1-(-1)^{k} \frac{\sin \alpha}{\alpha}\right],
$$


and therefore the non-dimensional $k$ th eigenvalue for the second family is given by

$$
\Lambda_{k}^{\mathrm{II}}=2 k^{2} \pi^{2} \alpha\left[\alpha-(-1)^{k} 2 \sin \alpha\right]+O(1), \quad k=1,2, \ldots
$$

In a similar manner, an approximation for non-dimensional eigenvalues can be derived for antisymmetric modes. In this case, the non-dimensional $k$ th eigenvalue corresponding to the first family is given by

$$
\Lambda_{k}^{\mathrm{I}}=(\pi \alpha)^{2}(2 k-1)^{2}, \quad k=1,2, \ldots
$$

while the non-dimensional $k$ th eigenvalue for the second family is given by

$$
\Lambda_{k}^{\mathrm{II}}=2 k^{2} \pi^{2} \alpha\left[\alpha+(-1)^{k} 2 \sin \alpha\right]+O(1), \quad k=1,2, \ldots
$$

It can be seen that the non-dimensional eigenvalues as functions of opening angle for a given symmetry do not intersect and it is therefore possible to arrange them in ascending order of frequency (see Table 1). This order is different for symmetric and antisymmetric modes and it defines the order in which the modes of a curved beam take one or another type of vibrational behaviour at large opening angle.

TABLE 1: Approximations in ascending order of frequency

\begin{tabular}{|c|cccccc|}
\hline Symmetric & $\Lambda_{1}^{\mathrm{II}}$ & $\Lambda_{1}^{\mathrm{I}}$ & $\Lambda_{2}^{\mathrm{II}}$ & $\Lambda_{2}^{\mathrm{I}}$ & $\Lambda_{3}^{\mathrm{II}}$ & $\Lambda_{3}^{\mathrm{I}}$ \\
\hline Antisymmetric & $\Lambda_{1}^{\mathrm{I}}$ & $\Lambda_{1}^{\mathrm{II}}$ & $\Lambda_{2}^{\mathrm{II}}$ & $\Lambda_{2}^{\mathrm{I}}$ & $\Lambda_{3}^{\mathrm{II}}$ & $\Lambda_{3}^{\mathrm{I}}$ \\
\hline
\end{tabular}




\subsection{Mode shapes at large opening angle}

In order to obtain expressions for the eigenfunctions up to a multiplying constant, we can set one of the coefficients $c_{i}$ in the expressions for normal displacements equal to 1 and estimate the remaining coefficients from boundary conditions. If we set $c_{3}=1$, then

$$
\begin{gathered}
c_{1}=\frac{-\beta_{2} f\left(\frac{\beta_{3} \alpha}{2}\right) g\left(\frac{\beta_{2} \alpha}{2}\right)+\beta_{3} f\left(\frac{\beta_{2} \alpha}{2}\right) g\left(\frac{\beta_{3} \alpha}{2}\right)}{\beta_{2} f\left(\frac{\beta_{1} \alpha}{2}\right) g\left(\frac{\beta_{2} \alpha}{2}\right)-\beta_{1} f\left(\frac{\beta_{2} \alpha}{2}\right) g\left(\frac{\beta_{1} \alpha}{2}\right)}, \\
c_{2}=\frac{-\beta_{3} f\left(\frac{\beta_{1} \alpha}{2}\right) g\left(\frac{\beta_{3} \alpha}{2}\right)+\beta_{1} f\left(\frac{\beta_{3} \alpha}{2}\right) g\left(\frac{\beta_{1} \alpha}{2}\right)}{\beta_{2} f\left(\frac{\beta_{1} \alpha}{2}\right) g\left(\frac{\beta_{2} \alpha}{2}\right)-\beta_{1} f\left(\frac{\beta_{2} \alpha}{2}\right) g\left(\frac{\beta_{1} \alpha}{2}\right)},
\end{gathered}
$$

where

$$
f \equiv\left\{\begin{array} { l l } 
{ \operatorname { c o s } } & { \text { symmetric modes } } \\
{ \operatorname { s i n } } & { \text { antisymmetric modes } }
\end{array} \quad g \equiv \left\{\begin{array}{ll}
\sin & \text { symmetric modes } \\
\cos & \text { antisymmetric modes }
\end{array}\right.\right.
$$

Firstly, consider the non-dimensional eigenvalue given by the second family of solutions (16) and (18). Substituting these expressions into (13), and the latter into the above expressions for $c_{1}$ and $c_{2}$, and taking the limit $\alpha \rightarrow \infty$ yields

$$
c_{1} \rightarrow 0, \quad c_{2} \rightarrow(-1)^{k+1},
$$

where $k$ is the mode index defined by $(16,18)$. Therefore, the form of normal displacements at large opening angle is, up to a constant,

$$
v \approx \begin{cases}\cos [\alpha(\bar{s}-1 / 2)] \cos [k \pi(\bar{s}-1 / 2)] & \text { for odd } k \\ \sin [\alpha(\bar{s}-1 / 2)] \sin [k \pi(\bar{s}-1 / 2)] & \text { for even } k\end{cases}
$$


for symmetric modes, and

$$
v \approx \begin{cases}\sin [\alpha(\bar{s}-1 / 2)] \cos [k \pi(\bar{s}-1 / 2)] & \text { for odd } k \\ \cos [\alpha(\bar{s}-1 / 2)] \sin [k \pi(\bar{s}-1 / 2)] & \text { for even } k\end{cases}
$$

for antisymmetric modes. We can see that the normal displacements in this case are highly oscillatory functions for which the spatial frequency of oscillation is proportional to the opening angle, modulated by a slowly varying function with frequency proportional to the mode index $k$ in the particular family of approximations. The tangential displacements in this case have similar structure and are of the same order of magnitude as the transverse displacements.

When the non-dimensional eigenvalue takes the values from the first family of solutions (15) and (17), the structure of the normal displacements is more complicated and generally all three terms in the expression (14) play significant roles. In this case it is convenient to consider the more clearly defined structure of the tangential displacements. The tangential displacements have the form

$$
\begin{aligned}
u= & b_{1} g\left[\beta_{1} \alpha(\bar{s}-1 / 2)\right]+b_{2} g\left[\beta_{2} \alpha(\bar{s}-1 / 2)\right] \\
& +b_{3} g\left[\beta_{3} \alpha(\bar{s}-1 / 2)\right]+\text { const }
\end{aligned}
$$

where

$$
b_{j}=\frac{c_{j}}{\beta_{j}} \quad j=1,2,3
$$


It is convenient in this case to set $c_{1}=1$, so that we have the following expressions for the remaining coefficients

$$
\begin{gathered}
c_{2}=\frac{-\beta_{3} f\left(\frac{\beta_{3} \alpha}{2}\right) g\left(\frac{\beta_{1} \alpha}{2}\right)+\beta_{1} f\left(\frac{\beta_{1} \alpha}{2}\right) g\left(\frac{\beta_{3} \alpha}{2}\right)}{\beta_{3} f\left(\frac{\beta_{3} \alpha}{2}\right) g\left(\frac{\beta_{2} \alpha}{2}\right)-\beta_{2} f\left(\frac{\beta_{2} \alpha}{2}\right) g\left(\frac{\beta_{3} \alpha}{2}\right)}, \\
c_{3}=\frac{-\beta_{1} f\left(\frac{\beta_{1} \alpha}{2}\right) g\left(\frac{\beta_{2} \alpha}{2}\right)+\beta_{2} f\left(\frac{\beta_{2} \alpha}{2}\right) g\left(\frac{\beta_{1} \alpha}{2}\right)}{\beta_{3} f\left(\frac{\beta_{3} \alpha}{2}\right) g\left(\frac{\left(\beta_{2} \alpha\right.}{2}\right)-\beta_{2} f\left(\frac{\beta_{2} \alpha}{2}\right) g\left(\frac{\beta_{3} \alpha}{2}\right)} .
\end{gathered}
$$

By substituting the expressions for $\Lambda$ from $(15,17)$ into $(13)$ and the latter into (21), (22) and (20), it is easy to show that

$$
b_{1}=O(\alpha), \quad b_{2}=O(1), \quad b_{3}=O(1) \quad \text { as } \quad \alpha \rightarrow \infty .
$$

The shape of the tangential displacements, up to the leading order, is given by

$$
u \approx \begin{cases}\alpha \sin [2 k \pi(\bar{s}-1 / 2)] & \text { for symmetric modes } \\ \alpha \cos [(2 k-1) \pi(\bar{s}-1 / 2)] & \text { for antisymmetric modes }\end{cases}
$$

where $k=1,2, \ldots$. It can be seen that the leading order part of the tangential displacements is a slowly varying function with a number of half-waves proportional to the mode index $k$ in a corresponding family of approximations. The transverse displacements in this case are of smaller order of magnitude than the tangential ones.

It can be seen that the structure of the mode shapes for the two families of approximations is essentially different. The tangential displacements of the modes from the first family are much larger than the radial ones. These 
modes can be seen as torsional modes of a macroscopic helix, with the torsion axis being the axis of the helix. The modes of the second family can be viewed as transverse ones with respect to the helical axis. It appears that these two different types of helical modes both originate from the flexural modes of a curved beam.

\section{$5 \quad$ Numerical results and discussion}

The numerical calculations in this section are produced using a collocation software for boundary-value ODEs "Colnew". In Figure 1, we plotted the numerical solution of equations (1-4) and small opening angle analytic approximations for the non-dimensional eigenvalue of the lowest antisymmetric and symmetric modes versus opening angle. It can be seen that the analytic approximations provide an accurate description of the region of decrease and subsequent rise of the non-dimensional eigenvalue and also give an accurate prediction of the position of its minimum. However, the vibrational behaviour for larger opening angle can not be predicted by this approximation. This is not surprising because the assumption that the mode shape remains without change becomes invalid at that stage.

In Figure 2, the numerical solutions for non-dimensional eigenvalues of the lowest three symmetric modes are shown together with large opening angle analytic approximations. It can be seen that the analytic approximations give an accurate prediction of non-dimensional eigenvalues for larger values 


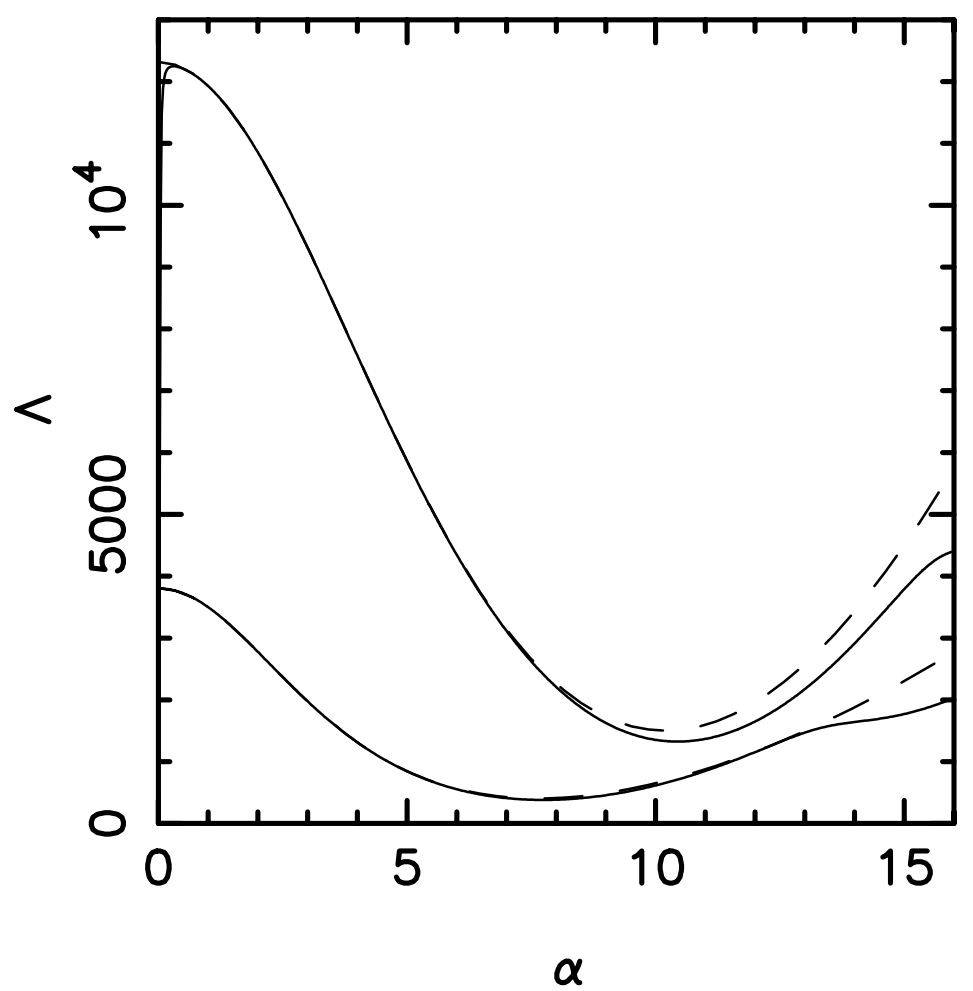

FIGURE 1: Non-dimensional eigenvalue as a function of opening angle for the lowest antisymmetric and symmetric modes. Analytic approximations are shown by dashed lines. 
5 Numerilo results and discussion

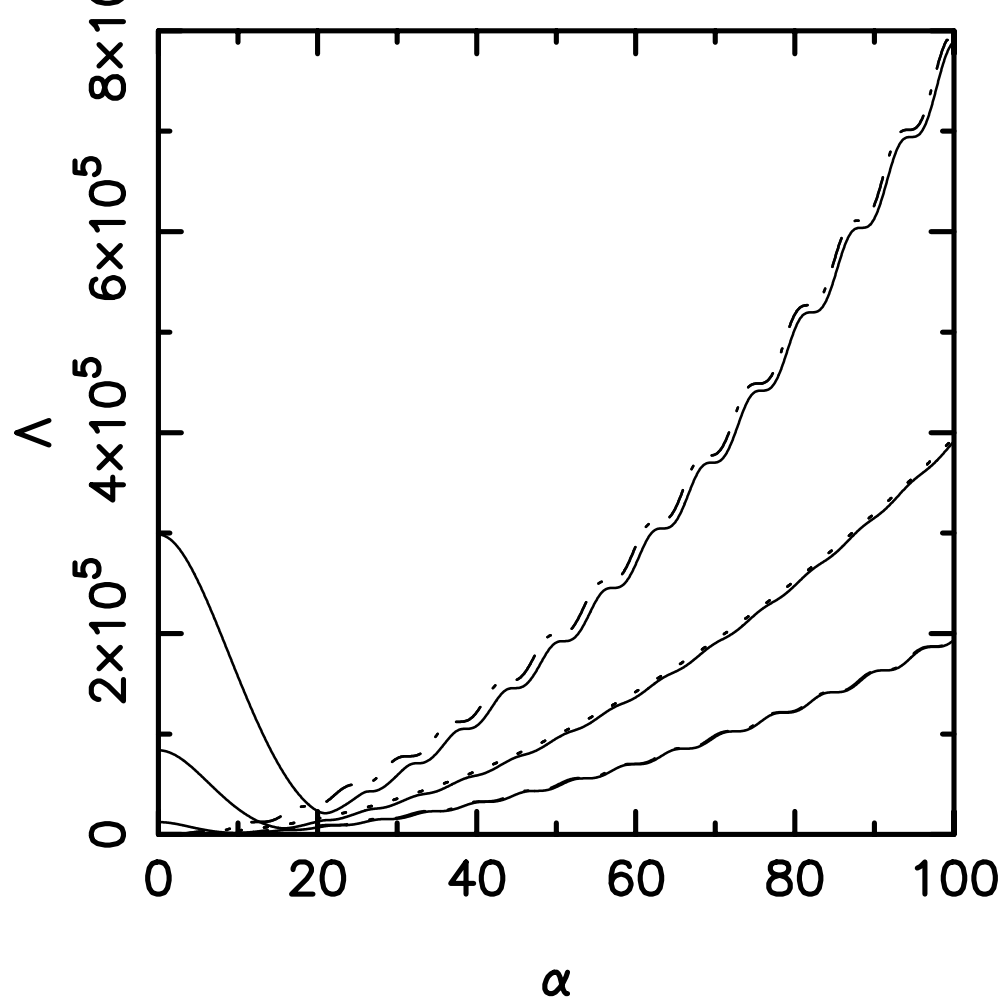

FiguRE 2: Numerical solution and large number of turns approximations for non-dimensional eigenvalue of symmetric modes. The analytic approximations of the first family are shown by dotted lines, while the second family is shown by dashed- dotted lines. 
of opening angle. The analytic approximations for the lowest two symmetric modes are almost indistinguishable from the exact numerical solution for a wide range of the opening angle. It is interesting to note that the second mode belongs to the first family of vibrational behaviour at large opening angle, while the lowest and the third modes belong to the second one. This is also clearly demonstrated in Figure 3, where the numerical solutions for the displacements of the three lowest symmetric modes are shown at a large value of opening angle. In the case of antisymmetric modes the order is different. The lowest mode belongs to the first family of vibrational behaviour, while the next two modes belong to the second one. It appears that the order of the modes (when arranged in ascending order of frequency) in the analytic approximation of a particular type of symmetry defines the order in which the modes of a curved beam of the same type of symmetry take one or another type of vibrational behaviour at large opening angle.

\section{Conclusions}

Analytic approximations are derived for the vibrational modes of a helix with small pitch in the plane normal to the helical axis, for the cases of small and large number of helical turns. It is shown that for a small number of helical turns the eigenvalues of all modes go through a minimum, which is closely related to the minimum of strain energy. For large numbers of helical turns, there are two families of asymptotic solutions for eigenvalues, 
a)

b)

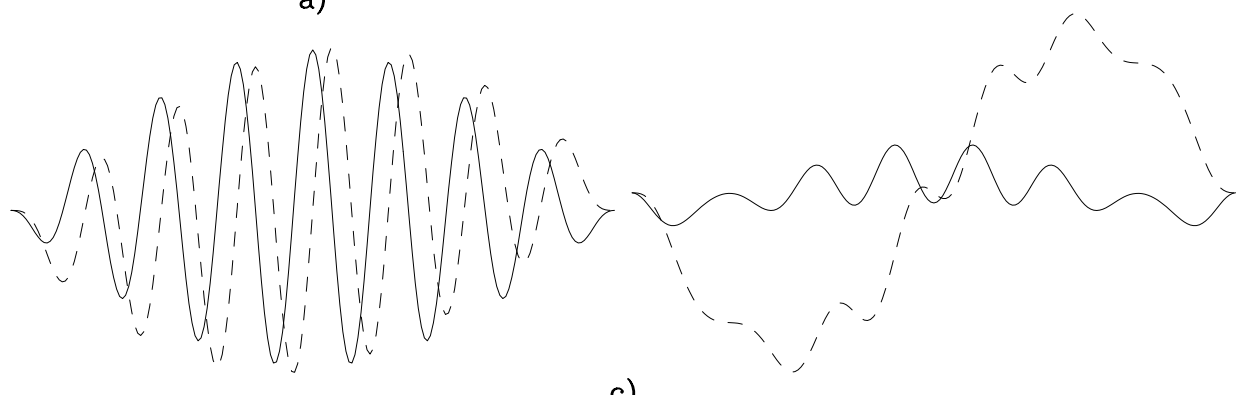

c)

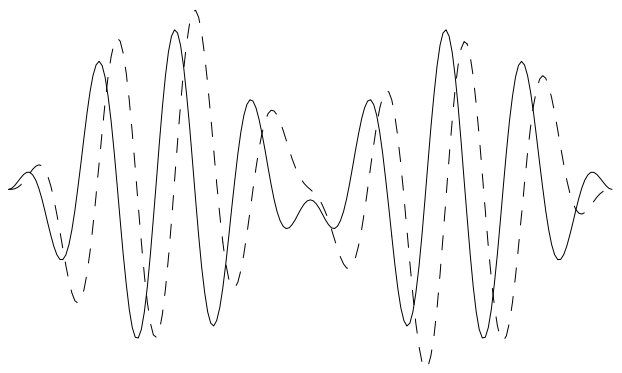

FiguRE 3: Eigenfunctions of the three lowest symmetric modes at $\alpha=50$. Transverse displacements are shown by solid lines, (a) first mode, (b) second mode, (c) third mode. 
which correspond to the two different types of helical modes. Both types of modes originate from the flexural modes of a uniformly curved beam and the order in which these latter modes develop into one or other of helical modes is established. Numerical simulations confirm the validity of our analytic approximations for a wide range of number of helical turns.

\section{References}

[1] A.E.H. Love. A Treatise on the Mathematical Theory of Elasticity, 4th edition. Dover Publications, New York, 1944. C1400

[2] Y. Kagawa. On the dynamical properties of helical springs of finite length with small pitch. Journal of Sound and Vibration, 8:(1)1-15, 1968. C1400, C1400, C1400

[3] L. Della Pietra and S. Della Valle. On the dynamic behaviour of axially excited helical springs. Meccanica, 17:31-43, 1982. C1400, C1400, C1400

[4] W. Jiang, W.K. Jones, T.L. Wang, and K.H. Wu. Free vibrations of helical springs. Journal of Applied Mechanics, 58:222-228, 1991. C1400, C1400, C1400 
[5] V. Yildirim and N. Ince. Natural frequencies of helical springs of arbitrary shape. Journal of Sound and Vibration, 204:311-329, 1997. C1400

[6] V. Yildirim. An efficient numerical method for predicting the natural frequencies of cylindrical helical springs. International Journal of Mechanical Sciences, 41:919-939, 1999. C1400

[7] T. Tarnopolskaya, F.R. de Hoog, N.H. Fletcher, and S. Thwaites. Asymptotic analysis of the free in-plane vibrations of beams with arbitrarily varying curvature and cross-section. Journal of Sound and Vibration, 196(5):659-680, 1996. C1400, C1402

[8] T. Tarnopolskaya, F.R. de Hoog and N.H. Fletcher. Low-frequency mode transition in the free in-plane vibration of curved beams. Journal of Sound and Vibration, 228(1):69-90, 1999. C1400, C1403

[9] T. Tarnopolskaya, F.R. de Hoog, A. Tarnopolsky, and N.H. Fletcher. Vibrations of beams and helices with arbitrarily large uniform curvature. Journal of Sound and Vibration, 228(2):305-332, 1999. C1402 